INPLASY PROTOCOL

To cite: Bhushan et al. Ultrasound-guided erector spinae plane block for postoperative analgesia in patients undergoing liver surgery: what we might know from a meta-analysis of Randomized control trials. Inplasy protocol 202210094. doi:

10.37766/inplasy2022.1.0094

Received: 18 January 2022

Published: 18 January 2022

Corresponding author: Sandeep Bhushan

dr.sdmch@aol.com

Author Affiliation: Chengdu Second People's Hospital.

Support: No founding.

Review Stage at time of this submission: Preliminary searches.

Conflicts of interest:

None declared.

\section{Ultrasound-guided erector spinae plane block for postoperative analgesia in patients undergoing liver surgery: what we might know from a meta-analysis of Randomized control trials}

Bhushan, S1; Xin, $\mathrm{H}^{2}$; Zongwei, $\mathrm{X}^{3}$.

Condition being studied: ESPB as an emerging regional technique has been well established in many surgeries, including reduce opioid demands, decrease pain score and improver sleep quality, etc. But, apply ESPB in liver surgery is limiting and remains uncertain, it is time to conduct one metaanalysis to reveal the performance of ESPB in liver surgery. Eligibility criteria: All published full-article RCTs comparing the analgesic efficacy of ESPB with control in adult patients undergoing any liver surgeries were eligible for inclusion. There were no language restrictions, Moreover, we also excluded case reports, non-RCT studies, incomplete clinical trials, and any trials used multiple nerve blocks. We also excluded any conference abstracts which could not offer enough information about the study design, or by data request to the author.

INPLASY registration number: This protocol was registered with the International Platform of Registered Systematic Review and Meta-Analysis Protocols (INPLASY) on 18 January 2022 and was last updated on 18 January 2022 (registration number INPLASY202210094).

\section{INTRODUCTION}

Review question / Objective: Liver surgeries (LSs) are associated with trauma and severe pain, adequate pain control is critical in patients undergoing major LSs. Although in the presence of advanced surgical techniques, including laparoscopic, minimal invasive open incision and robotic surgeries promotion, ensuring pain relief welfare is still of utmost importance in LSs. Regional anesthesia as one essential part of multi-modal anesthesia, including epidural analgesia 
(EA), paravertebral nerve block (PVB), transverse abdominal plane block (TAP), and local infiltration analgesia (LIA) have been widely applied in LSs. However, these regional analgesia techniques have their concerns, risks, and complications. EA and PVB can provide efficacious postoperative analgesia. Recent data suggested that both were difficult to perform, critics cite a reportedly high failure rate and relatively high complications ranging from pneumothorax to devastating spinal cord injury. Moreover, the coagulopathy of liver patients might limit the use of EA. The analgesic effect of TAP and LIA are often insufficient, neither reduce opioid consumption nor relieve visceral pain. Therefore, reasonable regional anaesthesia methods are needed in patients undergoing LH. ESPB as an emerging regional technique has been well established in various surgeries. Featured by its clinical, technical simplicity and wide dermatomal spread (T1 to L3), the newly defined fascial plane block (ESPB) has preferred by practitioners for its promising analgesia efficacy and safety across a variety of thoracic, breast, cardiac, lumbar, and hip surgical procedures. However, literature is scarce on the application of EPSB for postoperative analgesia in major liver surgeries, and randomized controlled studies are lacking and remaining controversial. Therefore, we conduct to perform a meta-analysis to determine the analgesic efficacy and safety of ESPB for liver surgery in adult surgical patients. We identified randomized controlled trials (RCT) comparing the ESPB with either nonblock care or other blocks, respectively. We hypothesized that the ESPB would be superior to non-block care but not inferior to other blocks. In terms of 24-hour postoperative resting pain scores and other analgesic makers, including opioid demand, first night sleep quality, postoperative vomiting/nausea, and blockrelated complications. ESPB has potential benefits in reducing postoperative pain score, opioid consumption and provides an alternative pain management strategy for patients.
Condition being studied: ESPB as an emerging regional technique has been well established in many surgeries, including reduce opioid demands, decrease pain score and improver sleep quality, etc. But, apply ESPB in liver surgery is limiting and remains uncertain, it is time to conduct one meta-analysis to reveal the performance of ESPB in liver surgery.

\section{METHODS}

Search strategy: We systematically searched PubMed, The Cochrane Library, Web of Science citation index, Embase from inception to November 2021 for RCTs meeting the listed inclusion criteria. Search strategy was combined of the terms detailed in the supplemental material. We also searched the grey literature by supplementary hand searching, for the ESPB is a newly regional anesthesia technique firstly introduced in 2016.

Participant or population: Patients undergoing all kind of liver surgeries with ASA class I-III, age 18-80 years old.

Intervention: Patients undergiong liver surgeryapplied with either ultrosund guided bilateral single injection of ESPB or bilateral continous injection of ESPBPatients in experimental group recived ultrasound-guided erector spinae plane block.

Comparator: Patients recived non-block or placebo.

Study designs to be included: We only included related RCTs.

Eligibility criteria: All published full-article RCTs comparing the analgesic efficacy of ESPB with control in adult patients undergoing any liver surgeries were eligible for inclusion. There were no language restrictions, Moreover, we also excluded case reports, non-RCT studies, incomplete clinical trials, and any trials used multiple nerve blocks. We also excluded any conference abstracts which could not offer enough information about the study design, or by data request to the author. 
Information sources: All published fullarticle RCTs comparing the analgesic efficacy of ESPB with control in adult patients undergoing any liver surgeries were eligible for inclusion. There were no language restrictions. Moreover, we also excluded case reports, non-RCT studies, incomplete clinical trials, and any trials used multiple nerve blocks. We also excluded any conference abstracts which could not offer enough information about the study design, or by data request to the author.

Main outcome(s): The primary outcome: postoperative $24 \mathrm{~h}$ resting pain scores.

Additional outcome(s): The secondary outcome: (1)postoperative 48-72hours resting and movement pain socres;(2) postoperative $24 \mathrm{~h}$ morphine consumption; (3) postoperative first night sleep quality; (4)postoperative vomiting ;(5)postoperative nausea;(6)postoperative shoulder pain; (7)other indications of patients recovery.

Data management: Data extraction to facilitate meta-analysis, medians, interquartile range (IQR), and range values were approximated into means and their corresponding SD as methods described by previous studies or as per Cochrane Handbook for Systematic Reviews of Interventions.

Quality assessment / Risk of bias analysis: Methodological quality assessment was independently assessed by $\mathrm{XH}$ and $\mathrm{BS}$, if any disagreements then resolved by a third author, according to the Cochrane Risk of Bias Tool and the Jadad score.

Strategy of data synthesis: We use Stata 15 vision and $R$ to manage data (the further step will continue)

Subgroup analysis: Due to the limiting RCT, we are not expected to do subgroup study between bilateral single injection of ESPB and bilateral continous injection of ESPB compare to control.
Sensitivity analysis: We use Stata 15 vision and $R$ to manage data (the further step will continue).

Language: English.

Country(ies) involved: China.

Keywords: Liver surgery; erecror spinae plane block; ultrasound-guided; postoperative alagesia; regional anesthesia.

Contributions of each author:

Author 1 - Sandeep Bhushan - Concept/ Design, Data analysis/interpretation, drafting article, Critical revision of article, Approval of article, Statistics, Data collection.

Author 2 - Huang Xin - Concept/Design, Data analysis/interpretation, drafting article, Critical revision of article, Approval of article, Statistics, Data collection.

Author 3 - Xiao zongwei - Concept/Design, Data analysis/interpretation, drafting article, Critical revision of article, Approval of article, Statistics, Data collection. 